\title{
Accretion disk instability revisited
}

\section{Transient dynamics of rotating shear flow}

\begin{abstract}
P. A. Yecko
Department of Astronomy, Columbia University, New York, NY, USA

e-mail: yecko@columbia.edu

Department of Physics and Astronomy, Arizona State University, Tempe, Arizona, USA

Received 11 May 2004 / Accepted 28 June 2004

Abstract. Accretion disk flow in a local Cartesian (or "shearing box") approximation is examined for viscous three-dimensional linear disturbances. Eigenvalue computations predict that the flow is asymptotically stable unless the rotation number falls in the range $0<$ Ro $<1 / 2$, in agreement with predictions of inviscid theory; Keplerian flow ( $\operatorname{Ro}=1 / q=2 / 3)$ is accordingly stable. Analysis of non-modal disturbances predicts large transient amplification factors, implying that the flow, although asymptotically stable, may be transiently unstable. Strong rotation, including Keplerian, two-dimensionalizes the system: the largest growth factors are found for disturbances which are uniform along the direction of the rotation axis and amplification occurs via the Orr mechanism, as in 2D shear flow. Amplification factors scale as $\mathrm{Re}^{2 / 3}$, implying very strong growth in actual disks. The implications of transient instability of rotating shear on disk turbulence are discussed.
\end{abstract}

Key words. accretion, accretion disks - instabilities - turbulence - hydrodynamics

\section{Introduction}

Astrophysical disks, in order to accrete, must transport angular momentum much more quickly than molecular viscosity permits. A turbulent viscosity could account for the greater transport, but brings with it the problems of the origin and nature of the turbulence. The identification of an instability is a natural theoretical route to possible turbulence, often realized by performing an eigenvalue analysis of linear perturbations made to a base flow. An incompressible inviscid fluid disk is stable, however, with respect to both centrifugal instability (Rayleigh's circulation criterion) and shear instability (Rayleigh's inflection point criterion and its variants). Disks with magnetic fields exhibit a linear magneto-rotational instability (Balbus \& Hawley 1991), and radial entropy gradients can lead to a linear baroclinic instability (Klahr \& Bodenheimer 2003) or, in a different arrangement, to a linear Rossby instability (a type of inflection point instability, Lovelace et al. 1999; Li et al. 2000). An intrinsic purely fluid mechanical instability or source of turbulence is, however, still of great interest. It is also now clear that instability predictions based on eigenvalues are really only relevant to the asymptotic fate of a flow. The idea that transient behavior cannot determine the stability of a flow has been all but abandoned, as we discuss in more detail below.

The sheared flow of an actual accretion disk includes many complicating effects, such as compressibility, stratification, and self-gravity, to name a few. In its most basic fluid mechanical form, however, the disk can be studied globally as an incompressible circular sheared flow. A local description, based on a point fixed within the disk, considers a portion of this flow evolving within a rotating reference frame. Accretion disk flow thus closely resembles curved and/or rotating shear flow, a configuration that has been studied extensively because of its applications in geophysics and turbomachinery.

Curvature and rotation are known to profoundly alter the basic stability properties of shear flow. In rotating shear, when the frame rotation and the flow vorticity vectors are antiparallel, instability can occur, taking the form of streamwise oriented vortices, also known as longitudinal rolls. This instability is due to Coriolis forces, as explained in Tritton (1992). An exactly analogous instability is found in circulating flows where it is caused instead by centrifugal forces. Taylor-Couette flow is an example of the latter type, where Rayleigh's circulation criterion gives the instability condition and the longitudinal rolls are known as Taylor vortices. For rotating flows, the analogous instability condition is that the total vorticity must be negative somewhere in the flow ${ }^{1}$ (Tritton 1992; Lezius \& Johnston 1975; Hart 1971; Bradshaw 1969). For a given shear flow, instability is therefore found only for a limited range of rotation rates. This rotational instability is independent of any viscous type instability, such as growing Tollmein-Schlichting waves, and

${ }^{1}$ An analogy can also be made to Boussinesq convection, in which the total vorticity plays the role of temperature gradient. 
of any shear instability, as described by Rayleigh's inflection point criterion.

Outside the limited range where rotation induces instability, it is considered to exert a stabilizing influence on shear. Rotationally stable shear may then become part of a class of flows which are predicted, by eigenvalue analysis, to be completely stable. Experience shows us that the prediction of instability by means of an eigenvalue analysis often fails: the linear perturbation problems for pipe Poiseuille and plane Couette flows yield no unstable eigenvalues, yet in practice both these flows are unstable and turbulent at moderate Reynolds numbers. Similarly, boundary layer flows and plane Poiseuille flows become unstable and turbulent below the critical Reynolds number predicted by eigenvalue analysis. While these flows may be nonlinearly unstable, the Reynolds-Orr theorem tells us that a linear mechanism is required for energy growth (Schmid \& Henningson 2000), so we should not have to adopt an entirely nonlinear approach to understand what destabilizes these flows.

The ability of many predicted stable flows to become turbulent, sometimes called bypass transition, has been linked to the presence of transiently growing disturbances in these flows. Support for this picture is found in the large predicted transient growth factors and by the prediction of features, such as streamwise vortices and streaks, that are common in turbulent and transitional flows. Transient growth may therefore be critical to understanding accretion disk flows, rotating laboratory shear flows such as those of Johnston et al. (1975) and numerical experiments, such as those of Bech \& Andersson (1997), where turbulence is seen despite predicted stability. Recently, transient growth has been extended to include a family of rotationally stable shear flows (Yecko \& Rossi 2004, hereafter YR).

The ability of disturbances in shear flows to grow transiently was first proposed by Kelvin (1887) and explored by Orr (1907); later, Ellingson \& Palm (1975), Landahl (1980) and Hultgren \& Gustavsson (1981) proposed mechanisms for this growth. Transient growth theory has taken shape more recently, see e.g., papers (Boberg \& Brosa 1988; Butler \& Farrell 1992; Trefethen et al. 1993; Reddy \& Henningson 1993), reviews, (Grossmann 2000; Reshotko 2001) and the monograph (Schmid \& Henningson 2000). For steady flows, transient growth can be related to the non-orthogonality, with respect to a suitable energy norm, of the eigenvectors of the linear evolution operator. This feature permits certain initial conditions to experience relatively large amplification $G(t)$ at intermediate times even for asymptotically stable flows. No instability is able to grow forever, so in this sense transient growth can be an equally important gauge of flow behavior as eigenvalue (asymptotic) analysis as long as growth is large enough to change the flow. Transient growth may therefore be more relevant than nonlinear instability to subcritical transition to turbulence. Schmid \& Henningson (2000) have offered a proof, based on theorems from linear algebra, that subcritical transition is possible only because the linear disturbance problem for shear involves a non-normal operator.

In the astrophysical literature, a form of transient growth was first advanced by Goldreich \& Lynden-Bell (1965) and applied to an accretion disk by Korycansky (1992). More recently,

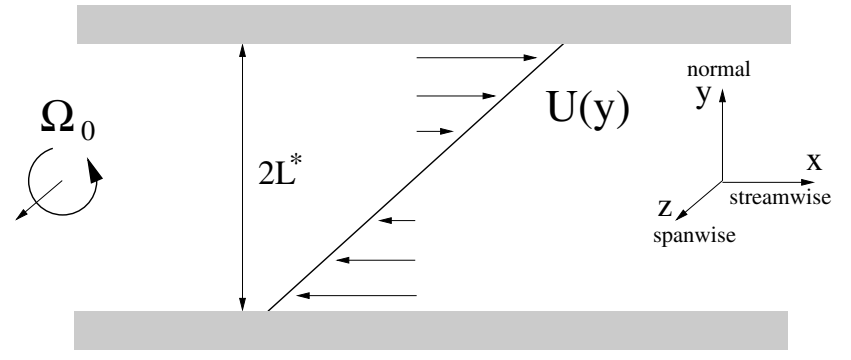

Fig. 1. Sketch of the rotating flow.

2D transient growth has been examined in a global disk model by Ioannou \& Kakouris (2001), and by Chagelishvili et al. (2003), who give a more complete connection between disk flow and examples from fluid mechanics. In a sequel, Tevzadze et al. (2003) extend this analysis to 3D perturbations.

The main purpose of this work is to compute transient growth properties in rotationally stabilized shear characteristic of Keplerian flow and to show the dependence of the growth factor on the Reynolds number. Another purpose of this work is to clarify the connection between transient and asymptotic studies of shear flow stability and stability studies of astrophysical disks, complementing the work of Longaretti (2002), Chagelishvili et al. (2003) and Tevzadze et al. (2003). The formulation here is designed to link the properties of accretion disk flow to those of a rotating shear flow, identifying key theoretical, laboratory and numerical results in the fluid mechanics literature. In regimes where rotation imposes modal stability, laboratory and numerical studies have noted that rotation also has a stabilizing effect on turbulence, by which it is meant that levels of turbulence are reduced. Turbulence is not eliminated, except at low Re, and for a given Ro, turbulence always returns as $R e$ is increased.

In the next section we consider a model base flow for an accretion disk in a local Cartesian approximation. Then we describe the methods of computing eigenvalues and transient growth. This is followed by results for asymptotic and transient stability and a discussion of several implications for disks.

\section{Base flow}

The angular velocity in an incompressible, vertically uniform disk without self-gravity can be described by $\Omega(r)=\Omega_{0}\left(r_{0} / r\right)^{q}$, where $q=3 / 2$ for Keplerian motion. In a locally Cartesian coordinate system, this flow can be approximated as linear shear in a uniformly rotating frame, viz:

$U(y)=q \Omega_{0} y=\frac{U^{*}}{L^{*}} y$,

an approximation known as the shearing box (Goldreich \& Lynden-Bell 1965; Narayan et al. 1987). In this work we consider the stability of the flow (1) subject to frame rotation of magnitude $\Omega_{0}$ around the axis normal to the flow plane. We use a coordinate system standard for shear flow, as shown in Fig. 1, differing from the shearing box (SB) convention such that $y=x_{\mathrm{SB}}$ and $x=-y_{\mathrm{SB}}$. The flow of a disk includes an accretion component; uniform accretion, for example, is described by $V(y)=-V^{*} \hat{y}$. Because $V^{*} / U^{*} \ll 1$ generally, the 
$V$ flow component is neglected here ${ }^{2}$. Finally, we place the flow in a channel, introducing walls at $y= \pm L^{*}$ co-moving with the flow there.

\section{Mathematical foundations}

In a frame rotating along the $z$-direction with a constant angular velocity $\Omega_{0}$, the dimensionless Navier-Stokes equations read

$\frac{\partial \boldsymbol{u}}{\partial t}+\boldsymbol{u} \cdot \nabla \boldsymbol{u}+2 \operatorname{Ro} \hat{z} \times \boldsymbol{u}=-\nabla p+\frac{1}{\operatorname{Re}} \nabla^{2} \boldsymbol{u} ; \quad \nabla \cdot \boldsymbol{u}=0$

where non-dimensionalization has been performed using the channel half-width $L^{*}$, velocity $U^{*}=U\left(L^{*}\right)$ and pressure scale $\rho\left(U^{*}\right)^{2}$. Two dimensionless numbers result, the Reynolds number $\operatorname{Re}=U^{*} L^{*} / v$, and the Rotation number ${ }^{3}$ Ro $=\Omega_{0} L / U^{*}$. Viscosity is kept here in order to show the parametric dependence of transient instability on Re.

The flow of Eq. (1) is an exact steady solution of Eq. (2) provided there is a base pressure $P(y)$ whose gradient balances the Coriolis force. Following standard methods, the base state velocity $\boldsymbol{U}=(U(y), 0,0)$ and pressure $P(y)$ are perturbed by adding an infinitesimal disturbance $\left(u^{\prime}, v^{\prime}, w^{\prime}\right)$ and $p^{\prime}$. The linearized equations of motion then appear:

$\frac{\partial u^{\prime}}{\partial t}+U \frac{\partial u^{\prime}}{\partial x}+v \frac{\mathrm{d} U}{\mathrm{~d} y}=-\frac{\partial p^{\prime}}{\partial x}+\frac{1}{\operatorname{Re}} \nabla^{2} u^{\prime}+2 \operatorname{Ro} v$

$\frac{\partial v^{\prime}}{\partial t}+U \frac{\partial v^{\prime}}{\partial x}=-\frac{\partial p^{\prime}}{\partial y}+\frac{1}{\operatorname{Re}} \nabla^{2} v^{\prime}-2 \operatorname{Ro} u$

$\frac{\partial w^{\prime}}{\partial t}+U \frac{\partial w^{\prime}}{\partial x}=-\frac{\partial p^{\prime}}{\partial z}+\frac{1}{\operatorname{Re}} \nabla^{2} w^{\prime}$

$\frac{\partial u^{\prime}}{\partial x}+\frac{\partial v^{\prime}}{\partial y}+\frac{\partial w^{\prime}}{\partial z}=0$

with the boundary conditions $u^{\prime}=v^{\prime}=w^{\prime}=0$ at $y= \pm 1$. We will refer to the coordinates $x, y, z$ (azimuthal, radial and disknormal directions) as the streamwise, normal and spanwise directions, respectively (see Fig. 1). Note that here the rotation number is related to the disk flow parameter $q$ via: $q=1 /$ Ro.

The linear perturbations can be assumed to satisfy a modal behavior in $x, z$, viz.

$\left(u^{\prime}, v^{\prime}, w^{\prime} ; p^{\prime}\right)=(u(y, t), v(y, t), w(y, t) ; p(y, t)) \mathrm{e}^{i(\alpha x+\beta z)}$,

where $\alpha, \beta$ are assumed to be purely real and we have dropped the use of primes on the perturbation quantities, without risk of confusion with the (unprimed) total quantities of Eq. (2).

By introducing the normal vorticity $\zeta(y, t)=i \beta u-i \alpha w$, the linear dynamics can be described by one equation governing normal velocity $v$ :

$$
\begin{gathered}
\left(\frac{\partial}{\partial t}+i \alpha U\right)\left(D^{2}-k^{2}\right) v-i \alpha\left(D^{2} U\right) v \\
+2 i \beta \operatorname{Ro} \zeta-\frac{1}{\operatorname{Re}}\left(D^{2}-k^{2}\right)^{2} v=0,
\end{gathered}
$$

${ }^{2}$ See YR for an analysis of the effect of $V$ on stability properties of rotating shear flow.

${ }^{3}$ In many applications, the Rossby number Ros $=1 /(2 \mathrm{Ro})$ is used instead; also, some authors define Ro to include a factor of 2. and a second equation governing normal vorticity $\zeta$ :

$\left(\frac{\partial}{\partial t}+i \alpha U\right) \zeta-2 \operatorname{Ro} i \beta v+i \beta(D U) v-\frac{1}{\operatorname{Re}}\left(D^{2}-k^{2}\right) \zeta=0$,

where $k^{2} \equiv \alpha^{2}+\beta^{2}, D \equiv \mathrm{d} / \mathrm{d} y$. Equations (8) and (9) are the rotationally modified Orr-Sommerfeld and Squire equations, respectively. The boundary conditions become

$v=D v=\zeta=0 \quad$ at $\quad y= \pm 1$.

A dynamical system is thus defined for $\boldsymbol{q}(\boldsymbol{y}, \boldsymbol{t})=$ $(v(y, t), \zeta(y, t))^{T}$.

\subsection{Asymptotic instability}

The focus of this work is the transient growth dynamics of the above problem, but as a jumping-off point we also examine the behavior of normal modes. The modal approach consists in recasting the problem into an eigenvalue problem by assuming $\boldsymbol{q}=\tilde{\boldsymbol{q}}(\boldsymbol{y}) \mathrm{e}^{-i \omega t}$ where $\tilde{\boldsymbol{q}}=(\tilde{v}, \tilde{\zeta})^{T}$ and $\omega=\omega_{r}+i \omega_{i}$ are complex. Clearly a mode having $\omega_{i}>0$ is unstable. Equations (8) and (9) can then be written

$i \omega \boldsymbol{M} \tilde{\boldsymbol{q}}=\boldsymbol{L} \tilde{\boldsymbol{q}}$

where

$\boldsymbol{M}=\left(\begin{array}{cc}D^{2}-k^{2} & 0 \\ 0 & 1\end{array}\right), \quad \boldsymbol{L}=\left(\begin{array}{cc}\mathcal{L}_{\mathrm{OS}} & \mathcal{R}_{\mathrm{OS}} \\ \mathcal{R}_{\mathrm{SQ}} & \mathcal{L}_{\mathrm{SQ}}\end{array}\right)$

with

$\mathcal{L}_{\mathrm{OS}}=i \alpha U\left(D^{2}-k^{2}\right)-i \alpha\left(D^{2} U\right)-\frac{1}{\operatorname{Re}}\left(D^{2}-k^{2}\right)^{2}$,

$\mathcal{L}_{\mathrm{SQ}}=i \alpha U-\frac{1}{\operatorname{Re}}\left(D^{2}-k^{2}\right)$,

$\mathcal{R}_{\mathrm{OS}}=2 i \operatorname{Ro} \beta, \mathcal{R}_{\mathrm{SQ}}=i \beta(D U)-2 i \operatorname{Ro} \beta$.

For linear shear, the $D^{2} U$ term in $\mathcal{L}_{\mathrm{OS}}$ is zero and will be dropped from now on. It should be emphasized that as a result of rotation, the Orr-Sommerfeld equation is explicitly coupled to the Squire equation (both $\mathcal{R}_{\mathrm{OS}}$ and $\mathcal{R}_{\mathrm{SQ}}$ are nonzero) while in the absence of rotation only the Squire equation is coupled, through $\mathcal{R}_{\mathrm{SQ}}$. In both cases, coupling occurs only for disturbances with $\beta \neq 0$. These equations together with the boundary conditions

$\tilde{v}=D \tilde{v}=\tilde{\zeta}=0 \quad$ at $y= \pm 1$,

define the eigenvalue problem to be solved. A spectral collocation algorithm developed in previous work (YR) is implemented, in which eigenfunctions are expanded using Chebyshev polynomials. The number $N$ of polynomials was generally $N=75$, although as many as $N=125$ were used to give improved accuracy in some calculations. Collocation points are the standard Gauss-Lobatto points. The eigenvalue problem (11) and boundary conditions (16) are then transformed into a corresponding $2 N \times 2 N$ matrix problem. To facilitate calculation, the eigenvalue problem (11) is actually written as

$i \omega \tilde{\boldsymbol{q}}=\mathcal{L} \tilde{\boldsymbol{q}}$ with $\mathcal{L} \equiv \boldsymbol{M}^{-1} \boldsymbol{L}$. 


\subsection{Transient instability}

Transient amplification calculations have also been implemented on the problem in Sect. 3.1 according to the singular value decomposition (SVD) method given by Reddy \& Henningson (1993). The main steps of the method are briefly described below; more details can be found in the references. First, a form of the kinetic energy norm (see Reddy et al. 1993):

$\|\boldsymbol{q}(\boldsymbol{t})\|_{E}^{2}=\frac{1}{2 k^{2}} \int_{0}^{L}\left(|D v|^{2}+k^{2}|v|^{2}+|\zeta|^{2}\right) \mathrm{d} y$

is used to measure the magnitude of a disturbance $q(t)$ at time $t$. At time $t$, one can then define

$G(t)=\sup _{\boldsymbol{q}(0) \neq 0} \frac{\|\boldsymbol{q}(\boldsymbol{t})\|_{E}^{2}}{\|\boldsymbol{q}(\mathbf{0})\|_{E}^{2}}=\left\|\mathrm{e}^{i \mathcal{L} t}\right\|_{E}^{2}$,

where $\boldsymbol{q}(\mathbf{0})$ is an initial disturbance and $\mathcal{L}$ is the linear operator defined in Eq. (17). This quantity $G(t)$ represents the maximum possible energy amplification at time $t$, optimized over all possible initial disturbances. The maximum or optimal growth is defined as $G_{\max }=\sup _{t \geq 0} G(t)$. The peak value $G_{\mathrm{P}}(\mathrm{Ro}, \mathrm{Re}) \equiv \sup _{\alpha, \beta} G_{\mathrm{max}}(\alpha, \beta, \mathrm{Ro}, \mathrm{Re})$ can also be computed.

The quantity $G(t)$ is obtained as follows. Assume that the eigenvalues of Eqs. (17) and (16) are sorted in order of decreasing imaginary part $\omega_{i}$. One can approximate $G(t)$ by computing the maximum possible energy amplification at time $t$, optimized over all possible initial combinations of the $K$ eigenfunctions associated with the first $K$ eigenvalues of Eq. (17). In this case, it is possible to transform the energy norm of the matrix exponential (19) to an ordinary 2-norm via the following equation

$\left\|\mathrm{e}^{i \mathcal{L} t}\right\|_{E}^{2} \approx\left\|\boldsymbol{F} \mathrm{e}^{-i \Lambda t} \boldsymbol{F}^{-1}\right\|^{2}$

where $\Lambda$ is a $K \times K$ diagonal matrix with the first $K$ eigenvalues $\omega_{1}, \omega_{2}, \ldots, \omega_{K}$ along the diagonal and $\boldsymbol{F}$ is obtained by Cholesky factorization of the $K \times K$ Hermitian Energy weight matrix $\mathcal{M}=\boldsymbol{F}^{\boldsymbol{H}} \boldsymbol{F}$ which is formed from the inner product of the $K$ eigenfunctions of Eq. (17), using the same inner product as the one defining the norm (18).

The approximation of Eq. (20) is a result of the finite number $K$ used in the expansion. As in YR, $K$ is chosen such that convergence is reached; in practice, $K \approx 75$ was found to be sufficient. Because $\mathcal{L}$ is non-normal, the response of the system is described by its largest singular value. The 2-norm on the RHS of Eq. (20) is thus evaluated using SVD, giving both $G_{\max }$ and the $K$ expansion coefficients of the disturbance associated with $G_{\max }$ (Reddy \& Henningson 1993; Butler \& Farrell 1992). Note that, since eigenfunctions have been expressed as an expansion in Chebyshev polynomials, all calculations are ultimately performed in terms of the Chebyshev expansion coefficients. In particular, the energy norm (18) and the weight matrix $\mathcal{M}$ are easily recast in terms of the expansion coefficients using the properties of Chebyshev polynomials and their derivatives (for details see Reddy et al. 1993).

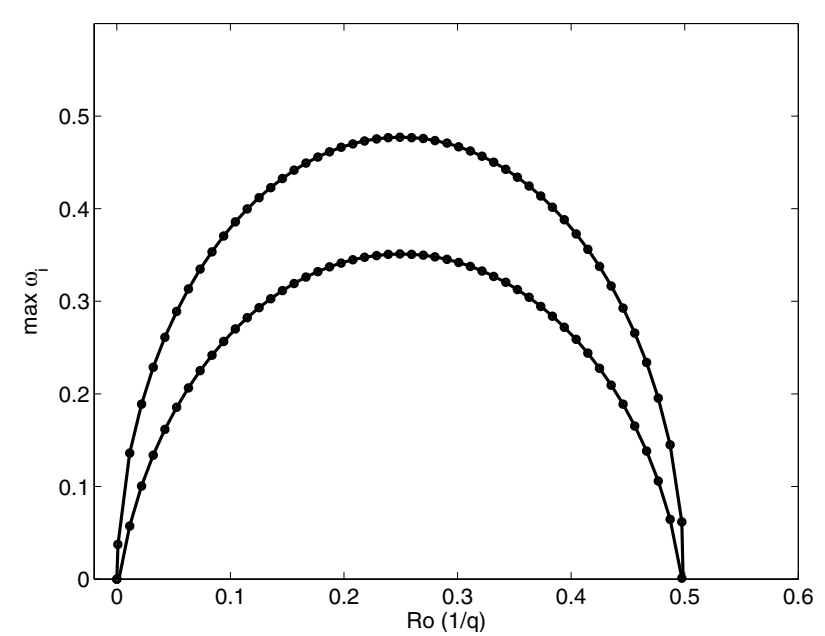

Fig. 2. Largest growth rates of the rotational mode at $\mathrm{Re}=150$ (lower curve) and $\operatorname{Re}=5000$ (upper curve); rotations $\operatorname{Ro}<0$ and $\operatorname{Ro}>1 / 2$ are called rotationally stabilized.

\section{Results}

\subsection{Modal stability properties}

It can be shown within inviscid theory (Hart 1971; Lezius \& Johnston 1975; Tritton 1992) that instability of a rotating parallel shear flow is described by the criterion that total vorticity must be negative somewhere in the flow ${ }^{4}$ i.e.

$\frac{1}{2 \operatorname{Ro}} \frac{\mathrm{d} U}{\mathrm{~d} y}>1$

although this criterion applies to viscous flows if $\operatorname{Re}$ is not too small (YR). Here $\mathrm{d} U / \mathrm{d} y=1$, implying that instability is restricted to the range $0<$ Ro $<1 / 2$. In Fig. 2 we show the largest exponential growth rate, $\left(\omega_{i}\right)_{\max }$, computed over a range of Ro and $\beta$ (we do not show for what $\beta$ this maximum occurs, but $\beta_{\max } \approx 1$ typically and $\alpha=0$ since the instability, longitudinal rolls, is known to be streamwise uniform). The predicted range of instability is recovered, while the growth rates at the lower Re value, to no surprise, are smaller in magnitude. Keplerian flow, for which Ro $=1 / q=2 / 3$, falls well within the stable region.

\subsection{Transient amplification}

The effect of rotation on transient growth is quantified through the analysis of amplification gain $G_{\max }(\alpha, \beta, \operatorname{Ro}, \operatorname{Re})$ as a function of streamwise wavenumber $\alpha$, spanwise wavenumber $\beta$, Reynolds number and Rotation number. In calculating $G_{\max }$ values, a fixed time interval $[0, T]$ is used during which the maximum growth is allowed to occur; the value of $T$ required to capture the maximum varies with $\mathrm{Re}$ and is adjusted accordingly in the calculations. We do not present any individual $G(t)$ curves here, instead showing only the maximum values as a function of $\alpha, \beta, \operatorname{Re}$, and Ro.

\footnotetext{
${ }^{4}$ Many works define $S=-2 \Omega / \frac{\mathrm{d} U}{\mathrm{~d} y}$ such that instability is found for $-1<S<0$; in cases where $\frac{\mathrm{d} U}{\mathrm{~d} y}$ is not constant, the local quantity $B=S(S+1)$ is used instead, such that $B<0$ indicates local instability.
} 

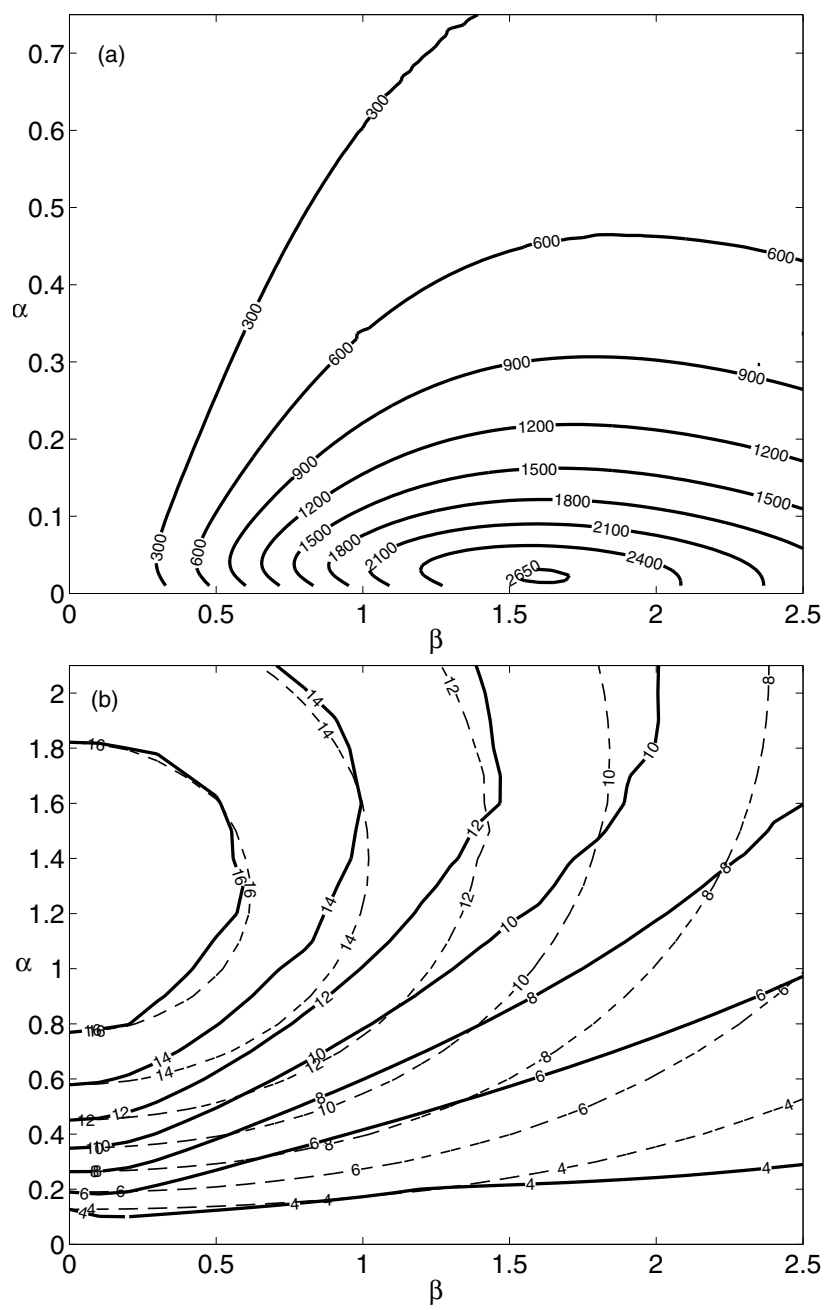

Fig. 3. a) Maximum transient growth factors $G_{\max }(\alpha, \beta)$ for nonrotating 3D Couette flow at $\mathrm{Re}=1500$; b) $G_{\max }(\alpha, \beta)$ for Keplerian $(\mathrm{Ro}=2 / 3)$ shear at $\mathrm{Re}=1500$ (solid curves) and for 2D non-rotating plane Couette flow (dashed).

Non-rotating Couette flow is known to display the following transient growth features (see, e.g., Schmid \& Henningson 2000): (i) the most amplified disturbances tend to be threedimensional with $\beta \neq 0$ and very small $\alpha \approx 0$; (ii) the peak amplification for large Reynolds number is proportional to the square of the Reynolds number, $G_{\max } \propto \mathrm{Re}^{2}$, a result that can be derived theoretically. For rotating Couette flow, the scaling $G_{\mathrm{P}} \propto \mathrm{Re}^{2}$ is not preserved as shown below. Instead, the scaling typical of 2D transient growth is found for strong rotation (see also YR). The only simple scaling relation that has been theoretically advanced for 2D transient growth, to our knowledge, is that of Chagelishvili et al. (2003) where a $\mathrm{Re}^{2 / 3}$ law is derived for disturbances of spatial Fourier harmonic type in a slightly different context. Below, we show our numerically determined scaling properties.

In Fig. 3 we compare the maximum transient growth factors $G_{\max }$ for rotating plane Couette flow (rpCf) to the standard result for non-rotating plane Couette flow (pCf) at $\mathrm{Re}=1500$. PCf exhibits its strongest growth for disturbances with weak streamwise variation: $\alpha=0.023, \beta=1.6$, as seen in Fig. 3a. $G_{\mathrm{P}}=2665$ here, in excellent agreement with published results (Butler \& Farrell 1992; Trefethen et al. 1993). The growth quantified in Fig. 3a is typically associated with oblique nearly streamwise-oriented vortices which, by moving regions of fluid normally, create high and low speed streaks.

This picture changes dramatically in the presence of strong rotation, as Fig. $3 \mathrm{~b}$ makes obvious. Rotation reduces the growth factors and also changes the character of the most growing disturbances, such that they have only streamwise variation (i.e. $\beta=0$ ). At this large value of Ro, rotation has imposed a Taylor-Proudman regime, two-dimensionalizing the disturbed flow. For comparison, the results of exactly 2D calculations for pCf are also shown, superimposed as dashed curves in Fig. 3b. At larger Ro these two sets of curves coincide better; conversely, the 3D behavior of Fig. 3a is approached at smaller Ro. In the shearing box approximation of disk flow, the characteristic length scale, fixed by the requirement that Keplerian flow be well approximated by linear shear, is small. To describe larger scales would involve some approximation of the curvature of Keplerian flow. This would, after nondimensionalization, lead to a smaller Ro and thus increasingly 3D transient growth; on yet larger scales, two-dimensionality would be again imposed by the global geometry of the disk. In actual disks, strict two-dimensionality is unrealistic; vertical stratification, for example, would serve to maintain a degree of three-dimensionality to the flow, in addition to supporting additional modes in the form of internal gravity waves. A more extensive analysis of transient growth over a range of Ro, showing the smooth transition from the 3D behavior, as in Fig. 3a, to approximate $2 \mathrm{D}$ behavior, as in Fig. 3b, has been performed in YR where we refer the interested reader. Tagger (2001) has pointed out that departure from a linear velocity profile is also needed to capture dynamical processes, such as Rossby waves, which rely on a vorticity gradient. The shearing box therefore lacks this generic and important dynamical aspect of shear in addition to bundling rotation and shear together into a fixed global value Ro $=2 / 3$, stymieing a broader study of the interplay of rotation and shear.

The most growing, or optimal disturbance at $\mathrm{Re}=1500$, Ro $=2 / 3$ is found in Fig. $3 \mathrm{~b}$ at $\alpha=1.2, \beta=0$ and has $G_{\mathrm{P}}=17.5$, occurring at $t_{\mathrm{P}}=10.1$. The reduction in $G_{\mathrm{P}}$ compared to the non-rotating case is dramatic: two orders of magnitude, but more relevant to accretion disks is the scaling of $G_{\mathrm{P}}$ with Re. This scaling is treated below.

Because the optimal disturbance at Ro $=2 / 3$ is spanwise uniform, we can focus on the dependence of $G_{\max }$ on $\mathrm{Re}$ and on the streamwise wavenumber $\alpha$. In Fig. 4 we show the maximum growth factors $G_{\max }$ in the $\alpha-\operatorname{Re}$ plane. The streamwise wavenumber of peak growth $\alpha_{\mathrm{P}}$ depends weakly on Re, as evident in the nearly vertical level curves of Fig. 4; for example, at $\operatorname{Re}=1000, \alpha_{\mathrm{P}}=1.2$ while at $\operatorname{Re}=10^{5}, \alpha_{\mathrm{P}} \approx 1$. An inspection of the spacing of the level curves suggests that $G_{\max } \propto \mathrm{Re}^{n}$. In Fig. 5 we collapse the data of Fig. 4, showing only the largest $G_{\max }$ values (i.e. $G_{\mathrm{P}}$ ) at each Re. The resulting trend corresponds closely to a $\mathrm{Re}^{2 / 3}$ scaling, as can be seen from the constructed slope-2/3 line. Such a scaling differs substantially from the $\mathrm{Re}^{2}$ scaling of $3 \mathrm{D}$ transient growth, but nevertheless implies increasingly large growth factors with Re, and potentially explosive growth in disks. 


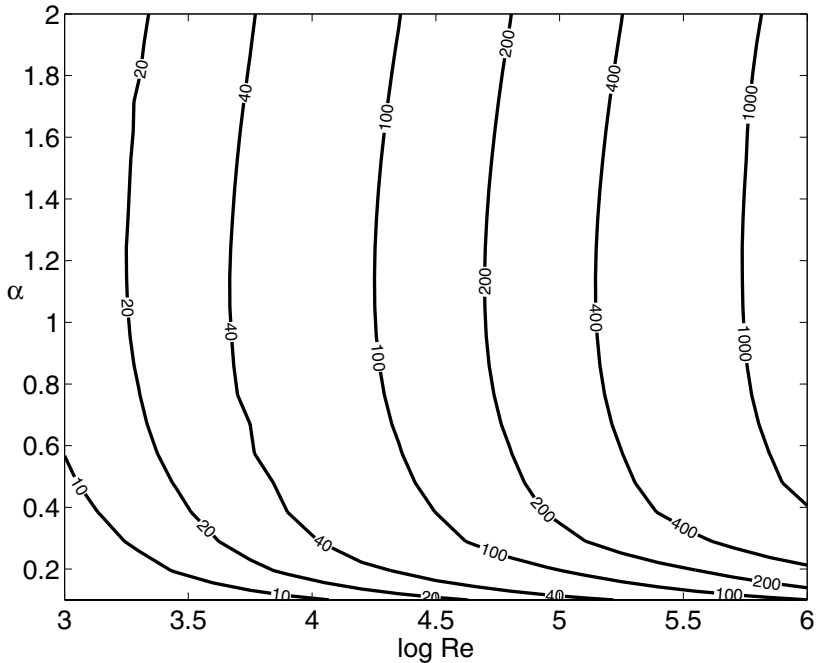

Fig. 4. Maximum transient growth factors $G_{\max }(\alpha, \operatorname{Re})$ for Keplerian $(\operatorname{Ro}=2 / 3)$ shear

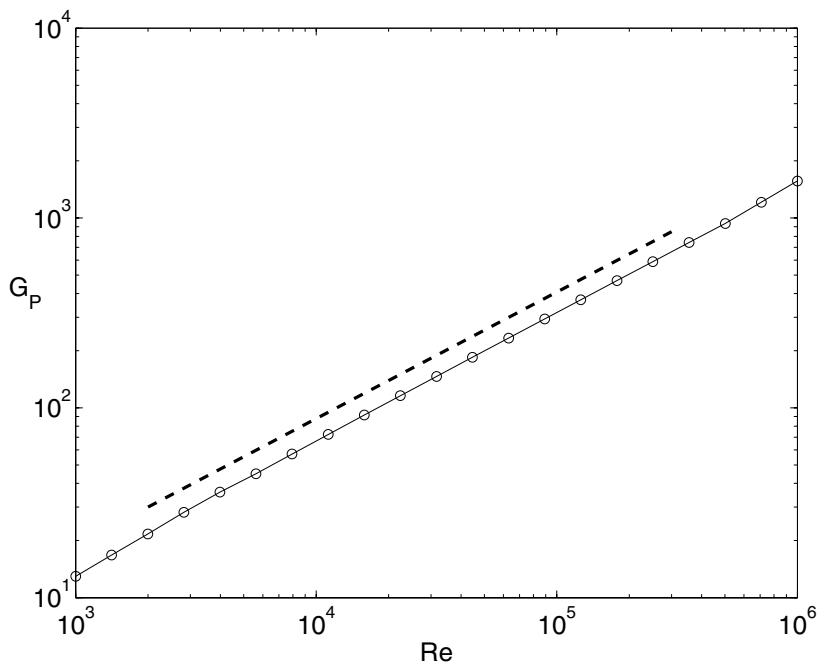

Fig. 5. Peak transient growth factors $G_{\mathrm{P}}$ as a function of Reynolds number for Keplerian $($ Ro $=2 / 3$ ) shear (solid line and points); $G_{\mathrm{P}} \propto \mathrm{Re}^{2 / 3}$ (dashed) for comparison.

Since 2D transient growth corresponds to the Orr mechanism (or vortex tilting, see Butler \& Farrell 1992 or Schmid \& Henningson 2000), we expect that the most amplified disturbances reflect this property. This is confirmed by Fig. 6, where we show the computed flow fields at $t=0$ and $t=t_{\mathrm{P}}$ of the optimal disturbance at $\operatorname{Re}=1500, \mathrm{Ro}=2 / 3$. The optimal disturbance at $t=0$ consists of flow arranged in opposition to the base shear, as shown in Fig. 6a. By $t=t_{\max }=10.11$, the shear has tilted the disturbance into an array of counter-rotating vortices spanning the channel, as seen in Fig. 6b. Because vorticity is conserved, velocities (and therefore energy) must increase for the large area vortices at $t_{\max }$, as explained in Butler \& Farrell (1992) (see also Orr 1907; Schmid \& Henningson 2000). Ioannou \& Kakouris (2001) have shown that such disturbances transport angular momentum outward in disks.
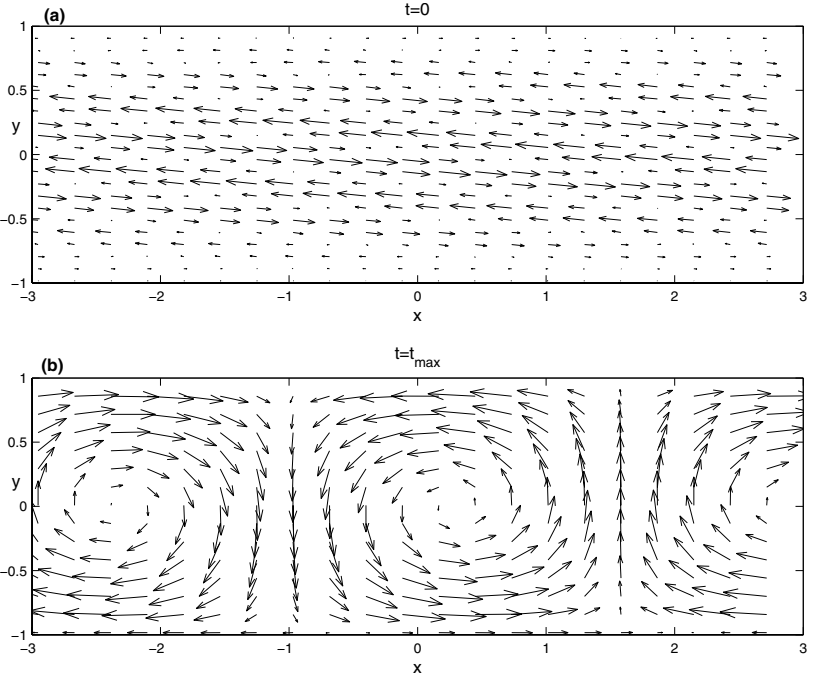

Fig. 6. The flow field $\boldsymbol{u}=(u, v, 0)$ of the optimal disturbance at $t=0 \mathbf{a}$ ); and at the time of maximum amplification, $t=t_{\max } \mathbf{b}$ ); at $\operatorname{Re}=1500, \operatorname{Ro}=2 / 3, \alpha=1.2$ and $t_{\max }=10.11$.

\section{Discussion}

\subsection{Reynolds averaging and nonlinear stability}

The stabilizing and destabilizing effects of rotation on shear turbulence have also been examined using Reynolds averaging formalisms (Johnston et al. 1975; Ferziger \& Shaanan 1976; Speziale 1989; Tritton 1992; also see Cambon \& Scott 1999, for a recent review that includes rotating turbulence). Reynolds averaged descriptions were developed with three-dimensional turbulent flows in mind and are known to fail for 2D turbulence (Tennekes \& Lumley 1972) and rotating turbulence (Tritton 1992). This is consistent with the well-known property of rotation to impose quasi two-dimensionality on flows, turbulent or laminar (Hopfinger 1989; see also Sect. 5.2). Nonlocal processes in quasi-2D and rotating turbulence are strong and generally require a two-point (non-local, typically integrodifferential or spectral) description (Lesieur 1990; Cambon \& Scott 1999). Reynolds averaging, on the other hand, is essentially local, involving correlations evaluated at a single point in space (corresponding turbulence models are consequently referred to as one-point models).

Reynolds averaged descriptions thus lead to immediate difficulties in the description of rotating turbulence. We point out several problems in the following. First, consider the equation for the evolution of turbulent energy; using standard tensor notation and summation convention, we write:

$\frac{\mathrm{d}}{\mathrm{d} t} \overline{q^{2}}=-\overline{u_{i} v_{j}} \frac{\mathrm{d} U_{i}}{\mathrm{~d} x_{j}}-\bar{\epsilon}$

where $\overline{q^{2}}=\overline{u_{i} u_{i}}$ and overbars denote averaging. Quantities $u_{i}$ here describe the turbulence, denoting fluctuations away from the mean state $\boldsymbol{U}$, such that $\boldsymbol{u}_{\text {total }}=\boldsymbol{U}+\boldsymbol{u}$, where $\boldsymbol{u}_{\text {total }}$ satisfies Eq. (2). The production term on the RHS of Eq. (22) represents work done by the Reynolds stress $\overline{u_{i} u_{j}}$, allowing fluctuations to exchange energy with the mean flow, while $\bar{\epsilon}$ represents viscous dissipation. Because Coriolis forces do not 
contribute to the energy, Eq. (22) is the same for rotating and non-rotating flows. Yet rotation is known to significantly affect turbulent energy; this conundrum is the first indication that the effects of rotation on turbulence are poorly captured by Reynolds stresses. Speziale (1989) has examined turbulence models based on Reynolds stresses, and shown that these fail for rotating flows because they are inconsistent with the rotating Navier-Stokes equations. Positivity of the production term, which indicates that turbulent energy is being produced, merely restates Rayleigh's Inflection Point criterion for shear instability of the mean flow. Furthermore, when rotation destabilizes the flow (as in Sect. 4.1, Fig. 2) the production term may be negative, implying that turbulence is suppressed even while vigorous turbulence is seen in experiments (see Sect. 5.2). Local correlations, such as $\overline{u v}$, therefore give unreliable descriptions of turbulence - including turbulent momentum transport - in the presence of rotation.

Balbus et al. (1996) studied fluctuations away from Keplerian flow (not a mean flow), applied averaging and identified the averaged correlations with Reynolds stresses and the fluctuation energy with that of turbulence. The failures discussed above have shown that such averaged quantities are not good diagnostics of rotating turbulence. Balbus et al. (1996) have correctly pointed out that Keplerian turbulence differs fundamentally from (3D) shear turbulence, also correctly attributing this to rotation, but have failed to recognize that Keplerian turbulence is a precise example of rotating turbulence, for which conventional descriptions are known to fail.

One may, in an approach similar to that above, study the stability of a flow by examining the energetics of disturbances away from a laminar base flow; this is sometimes called the energy method. The equations governing component energies are formed analogously to Reynolds stress equations, except averaging need not be performed (see Drazin \& Reid 1981; or Schmid \& Henningson 2000, for the more details). The evolution of total fluctuation energy $E_{V}=\int_{V} u_{i} v_{j}$ is found by integrating the component equations over the fluid volume and summing, giving:

$\frac{\mathrm{d} E_{V}}{\mathrm{~d} t}=-\int_{V} u_{i} u_{j} \frac{\partial U_{i}}{\partial x_{j}} \mathrm{~d} V-\frac{1}{\operatorname{Re}} \int_{V} \frac{\partial u_{i}}{\partial x_{j}} \frac{\partial u_{i}}{\partial x_{j}} \mathrm{~d} V$.

Equation (23) is known as the Reynolds-Orr equation. Nonlinear terms (cubic and higher in $u_{i}$ ) have integrated out and rotation terms from $u_{1}$ and $u_{2}$ components have canceled out, as for Eq. (22). The second term on the RHS of Eq. (23) represents viscous dissipation and always reduces the energy. The first term on the RHS represents the transfer of energy between the fluctuations and the background flow, as in Eq. (22). If this term is positive and large enough to overcome the dissipation, instability is possible. Positivity of the first RHS term in Eq. (23) is equivalent to a restatement of Rayleigh's Inflection Point criterion for inviscid shear ${ }^{5}$. The energy method in this context reduces to the predictions of linear theory (see Drazin \& Reid 1981; also Richard 2003); to extrapolate these prediction to turbulence would be unjustified.

\footnotetext{
${ }^{5}$ For viscous shear the first term on the RHS of Eq. (23) can also explain Tollmein-Schlichting type instability which can occur for flows where $\mathrm{d} U / \mathrm{d} y>0$ everywhere.
}

Finally, we re-emphasize that certain initial conditions can still grow to large amplitude transiently, as we have shown by direct computation of the transient energy (19), even for flows that are asymptotically stable. Although seeming to contradict Eq. (23), the apparent conflict is illusory, as discussed in detail in Schmid \& Henningson (2000).

\subsection{Relevant laboratory and numerical results}

Many laboratory experiments on rotating shear look at channel, duct or wake flows where the base flow vorticity and rotation are parallel $($ Ro $<0)$ in one region of the flow and anti-parallel $($ Ro $>0)$ in another. In the parallel region, Ro obviously falls in the stabilized regime (see Fig. 2), making it possible to simultaneously observe destabilized and stabilized shear in the same experiment. For stronger rotation, e.g. Ro $>0.5$, the entire flow can be stabilized. In such experiments (see, e.g., MacFarlane et al. 1998; Bidokhti \& Tritton 1992; Watmuff et al. 1985; Koyama et al. 1979; Johnston et al. 1975) the rotationally stabilized parts of the flow show reduced levels of turbulence, or laminarized flow. When rotation is increased at fixed Re, further reduction - including apparent disappearance - of turbulence is observed. Yet turbulence returns as $\mathrm{Re}$ is increased at fixed Ro, as seen in the experiments of Johnston et al. (1975). The laboratory data show that for any Ro there is a value of Re above which the flow is fully turbulent. Another, smaller value of Re marks the point below which the completely laminar state is found - see, for example, Fig. 10 of Johnston et al. (1975) or its reproduction in Hopfinger (1989).

Recent numerical simulations of rotating shear have also revealed that turbulence is always found at large enough Re. Bech \& Andersson (1997) performed low Re direct numerical simulations (DNS), finding that turbulence in linear shear is completely lost at $\operatorname{Re}=1300$ when $\mathrm{Ro}=1$ (i.e. more stable than Keplerian rotation). Generally, however, they observe that the stable regime is accompanied only by a reduction of the turbulence, pointing out that turbulence should always be found at higher Re since complete suppression of turbulence should only occur for Ro $=\infty$ (Greenspan 1968). Lamballais et al. (1995) performed DNS of rotating channel flow at $\mathrm{Re}=3750$ finding reduced, but not eliminated, turbulent energy in the stabilized region. Pallares \& Davidson (2000) used large eddy simulations (LES) at moderate Reynolds number $(\operatorname{Re} \approx 3000)$ to model flows in a duct for a wide range of rotations. Turbulence, considerably reduced in the stabilized regime, was never eliminated. More recently, Reif \& Andersson (2003) simulate large Reynolds number rotating duct flow and find that, though reduced in intensity, turbulence persists in the stabilized regime.

The reduction of turbulence seen in laboratory and numerical experiments and the failures of Reynolds averaged descriptions are indications that rotation is, as expected, imposing a quasi-2D Taylor Proudman regime on the flow. Stabilized regions are seen to exhibit negative turbulent energy production, where turbulence feeds the mean flow: a common feature of quasi-2D and rotating turbulence. These flows thus exhibit a complex admixture of $3 \mathrm{D}$ and rotationally enforced 
quasi-2D turbulence. (Interestingly, the rotationally destabilized side shows a superposition of typical 3D streaky shear turbulence and the longitudinal rolls of the rotational instability of Sect. 4.1.)

Quasi-2D turbulence is prevalent in geophysical problems, in the form of geostrophic (or quasi-geostrophic) turbulence, where two-dimensionality is imposed by a combination of rotation and stratification; accretion disks are subject to these same influences. When studying geophysical flows, quasi-2D is often assumed at the outset, but in this study we have begun by considering 3D flows and allowed rotation to lead us to the quasi-2D state. In this way we have also sampled the turbomachinery literature.

Recall that 2D turbulence lacks vortex stretching along the direction of the rotation axis, thereby conserving vorticity and exhibiting a reverse energy cascade to larger scales while cascading enstrophy (squared vorticity) to small scales, where it can be dissipated. Within this framework, it is common to see the formation of long-lived structures, including coherent vortices and jets. Quasi 2D and rotating turbulence exhibit the same behavior in approximation. It is known (Rhines 1979) that turbulence in these cases is best described directly in terms of its vorticity, which experiences a down-gradient turbulent transport. In a Keplerian disk, the vorticity $\zeta=\frac{1}{2} r^{-3 / 2}$, implying outward angular momentum transport. One physical process (not the only one) that mediates the inverse energy cascade is vortex merger, a highly non-local event that is not captured by local averaging schemes commonly used in turbulence descriptions, including the Reynolds stress formalisms. The simulations of Reif \& Andersson (2003) showed this in practice by directly comparing a Reynolds averaging approach to DNS while Salhi \& Cambon (1997) reached a similar conclusion analyzing DNS and LES results in the context of several turbulence models, including rapid distortion theory.

Several studies have shown (Dubrulle \& Valdetarro 1992; Longaretti 2002; Richard \& Davis 2004) or verified (Klahr \& Bodenheimer 2003) that the turbulent dynamics of disks is quasi-2D, transporting angular momentum outward. The implications of quasi-2D turbulence for the presence of coherent long-lived vortices has been examined by Bracco et al. (1999), Godon \& Livio (1999), Li et al. (2001) and Klahr \& Bodenheimer (2003).

\subsection{Numerical issues}

The numerical values presented in the previous section become inaccurate when $\alpha \mathrm{Re}$ is large, accounting for the wiggly features seen in parts of Fig. 3. The origin of the numerical error lies in the difficulty in computing accurate eigenvalues and eigenfunctions at large $\alpha$ Re. In Fig. 7 we show the spectrum of eigenvalues associated with the eigenfunctions that were used to obtain the optimal disturbance of Fig. 6, also associated with the peak value $G_{\mathrm{P}}$ of Fig. $3 \mathrm{~b}$. To get accurate $G$ values and optimal disturbance properties, it was necessary to include eigenfunctions at the confluence of the three eigenvalue branches, plus a number of those in the vertical branch below and, naturally, that these functions and values be accurate. Too many

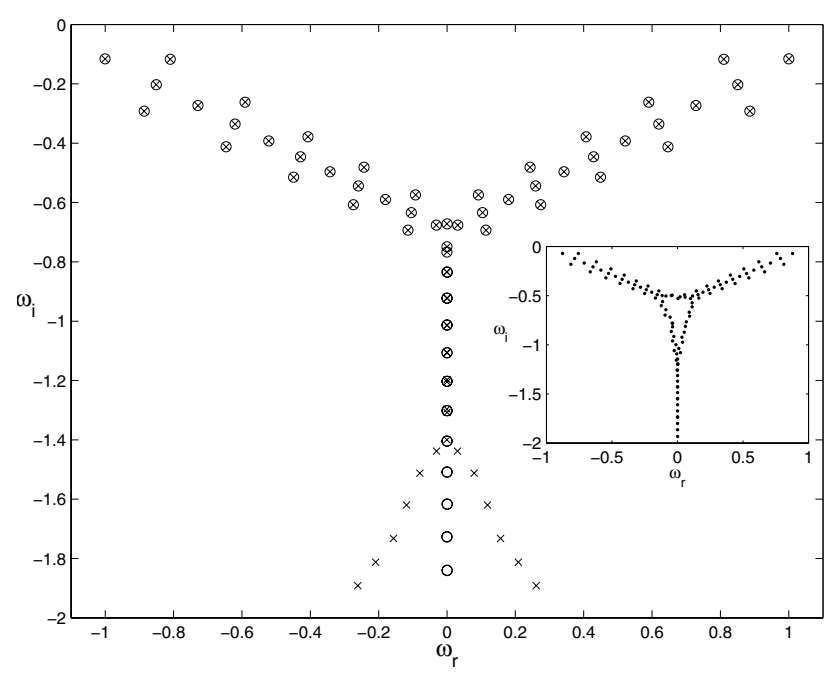

Fig. 7. Eigenvalue spectrum used to construct the optimal disturbance with $\operatorname{Re}=1500$, Ro $=2 / 3$ using $N=80$ polynomials (open circles) and $N=70$ polynomials (x marks), for which numerical error leading to lower branch splitting is seen; inset: numerical error at the confluence point at $\mathrm{Re}=4500$ resulting from too many polynomials, $N=140$.

Chebyshev polynomials exacerbates round-off error and leads to poor eigenvalues at the confluence point, as shown in the inset figure; too few leads to a downward splitting of the vertical branch, as illustrated in Fig. 7, the splitting point occurring at larger $\omega_{i}$ as $N$ decreases. These effects are well documented properties of Chebyshev methods, which in general tend to give the most accurate results for shear flow problems (see Dongarra et al. 1996).

If transient instability plays an important role in the turbulence of rotating shear flows, as this work suggests, computations of these flows must take place at high enough Re to exploit the $\mathrm{Re}^{2 / 3}$ scaling, and must have sufficient accuracy at high Re to resolve a substantial subset of the eigenfunction spectrum with $\omega_{i}>-\alpha$. Umurhan \& Regev (2004) have performed numerical simulations of disk flow targeting the evolution of transient growth. In a related study (Criminale et al. 1997), direct numerical solutions of the transient dynamics of pCf were able to reproduce all known stability data, but also found strong sensitivity to the form of the initial conditions, viz.: initial perturbations lacking normal vorticity gave inaccurate growth factors and unrealistic flow properties. Simulation of rpCf should be even more numerically challenging since comparable growth factors are found at larger Re.

\section{Conclusions}

In this work we have computed stability properties of 3D disturbances in a model accretion disk flow, focusing on the properties of transient growth in regimes where an eigenvalue analysis predicts asymptotic stability. Keplerian flow, with Rotation number $\operatorname{Ro}=2 / 3$, is rotationally stable, falling well outside the computed unstable range: $0<$ Ro $<1 / 2$. Strong rotation is shown to two-dimensionalize the most amplified transient disturbances, which therefore show streamwise variation $(\alpha \approx 1)$ but which are uniform along the rotation axis $(\beta=0)$. 
Two-dimensionality is consistent with the Taylor-Proudman regime associated with rotating flow. We have shown that peak growth factors scale as $\mathrm{Re}^{2 / 3}$ for large Ro (or 2D) flow over a wide range of Reynolds numbers. Disks, because of their astronomically large Reynolds numbers, would show enormous transient growth. Transient growth then becomes a viable route to turbulence, as it is now believed to be for many non-rotating flows. Because transient growth can occur in flows that are stable to rotational and shear instabilities, it provides a means to create fluctuation energy in those flows.

We emphasize that the transient growth mechanism is linear and that, according to the Reynolds-Orr theorem, a linear mechanism is required for instability in any flow, even flows predicted by eigenvalue analysis to be stable. This is important in light of previous claims (see Balbus et al. 1996) that any high Reynolds number instabilities are necessarily nonlinear. On the the other hand, while transient growth provides a powerful linear instability mechanism at high Reynolds numbers, additional effects - most likely nonlinear - are needed to exaplin the full development of transient instability, including its role, if any, in the transition to turbulence. These critical issues are subjects of future work.

Finally, we have discussed these results in the context of laboratory experiments and numerical simulations of shear subject to rotation, in particular the implications of the stability properties of rotation on turbulent rotating shear flows and their nature as quasi $2 \mathrm{D}$ turbulent flows.

Acknowledgements. I thank Sumner Starrfield for his hospitality and the ASU Physics \& Astronomy Department for providing resources.

\section{References}

Balbus, S., \& Hawley, J. 1991, ApJ, 376, 214

Balbus, S., Hawley, J., \& Stone 1996, ApJ, 467, 76

Bech, K. H., \& Andersson, H. I. 1997, J. Fluid Mech., 347, 289

Bidokhti, A. A., \& Tritton, D. J. 1992, J. Fluid Mech., 241, 469

Boberg, L., \& Brosa, U. 1988, Z. Naturforschung, 43a, 697

Bracco, A., Provenzale, A., Spiegel, E. A., \& Yecko, P. 1999, in Theory of Black Hole Accretion Disks (Cambridge University Press)

Bradshaw, P. 1969, J. Fluid Mech., 36, 177

Butler, K. M., \& Farrell, B. F. 1992, Phys. Fluids A, 4(8), 1637

Cambon, C., \& Scott, J. F. 1999, ARFM, 31, 1

Chagelishvili, G. D., Zahn, J.-P., Tevzadze, A. G., \& Lominadze, J. G. 2003, A\&A, 402, 401

Criminale, W. O., Jackson, T. L., Lasseigne, D. G., \& Joslin, R. D. 1997, J. Fluid Mech., 339, 55

Dongarra, J. J., Straughan, B., \& Walker, D. W. 1996, Appl. Num. Math., 22, 399

Drazin, P. G., \& Reid, W. H. 1981, Hydrodynamic stability (Cambridge University Press)

Dubrulle, B., \& Valdettaro, L. 1992, A\&A, 263, 387

Ellingson, T., \& Palm, E. 1975, Phys. Fluids, 18(4), 487

Ferziger, J. H., \& Shaanan, S. 1976, Phys. Fluids, 19(4), 596
Godon, P., \& Livio, M. 1999, ApJ, 523, 350

Goldreich, P., \& Lynden-Bell, D. 1965, MNRAS, 130, 125

Greenspan, H. 1968, Theory of Rotating Fluids (Cambridge University Press)

Grossmann, S. 2000, Rev. Mod. Phys., 72(2), 603

Hart, J. E. 1971, J. Fluid Mech., 45, 341

Hopfinger, E. J. 1989, Theoretical and Applied Mechanics, Proc. IUTAM, ed. P. Germain, M. Piau, \& D. Caillerie (Elsevier)

Hultgren, L. S., \& Gustavsson, L. H. 1981, Phys. Fluids, 24, 1000

Ioannou, P. J., \& Kakouris, A. 2001, ApJ, 550, 931

Johnson, J. A. 1963, J. Fluid Mech., 17, 337

Johnston, J. P., Halleen, R. M., \& Lezius, D. K. 1972, J. Fluid Mech., 56,533

Lord Kelvin (W. Thompson) 1887, Philos. Mag., 24, 188

Klahr, H. H., \& Bodenheimer, P. 2003, ApJ, 582, 869

Korycansky, D. G. 1992, ApJ, 399, 176

Koyama, H., Masuda, S., Ariga, I., \& Watanabe, I. 1979 Trans. ASME J. Eng. Power, 101, 23

Lamballais, E., Lesieur, M., \& Métais, O. 1995, Int. J. Heat Fluid Flow, 17, 324

Landahl, M. T. 1980, J. Fluid Mech., 98, 243

Lesieur, M. 1990, Turbulence in Fluids, 2nd ed. (Kluwer)

Lezius, D. K., \& Johnston, J. P. 1975, J. Fluid Mech., 77, 153

Li, J., Finn, J. M., Lovelace, R. V. E., \& Colgate, S. A. 2000, ApJ, 533, 1023

Li, J., Colgate, S. A., Wendroff, B., \& Liska, R. 2001, ApJ, 551, 874

Longaretti, P. 2002, ApJ, 576, 587

Lovelace, R. V. E., Li, H., Colgate, S. A., \& Nelson, A. F. 1999, ApJ, 513, 805

Macfarlane, I., Joubert, P. N., \& Nickels, T. B. 1998, J. Fluid Mech., 373,1

Narayan, R., Goldreich, P., \& Goodman, J. 1987, MNRAS, 228, 1

Orr, W. M. F. 1907, Proc. R. Irish Acad. A, 27, 9

Pallares, J., \& Davidson, L. 2000, Phys. Fluids, 12(11), 2878

Reif, B. A. P., \& Andersson, H. I. 2003, J. Turbulence, 4, 1

Rhines, P. B. 1979, Ann. Rev. Fluid Mech., 11, 401

Richard, D. 2003, A\&A, 408, 409

Richard, D., \& Davis, S. S. 2004, A\&A, 416, 825

Reddy, S. C., \& Henningson, D. S. 1993, J. Fluid Mech., 252, 209

Reddy, S. C., Schmid, P. J., \& Henningson, D. S. 1993, SIAM J. Appl. Math., 53(1), 15

Reshotko, E. 2001, Phys. Fluids, 13(5), 1067

Salhi, A., \& Cambon, C. 1997, J. Fluid Mech., 347, 171

Schmid, P. J., \& Henningson, D. S. 2000, Shear Flow Instability (Springer-Verlag)

Speziale, C. G. 1989, Theor. Comp. Fluid Dyn., 1, 3

Tagger, M. 2001, A\&A, 380, 750

Tevzadze, A. G., Chagelishvili, G. D., Zahn, J.-P., \& Lominadze, J. G. 2003, A\&A, 407, 779

Tennekes, \& Lumley 1972, A First Course in Turbulence (MIT Press)

Trefethen, L. N., Trefethen, A. E., Reddy, S. C., \& Driscoll, T. A. 1993, Science, 261, 578

Tritton, D. J. 1992, J. Fluid Mech., 241, 503

Umurhan, O. M., \& Regev, O. 2004, A\&A, submitted

Watmuff, J. H., Witt, H. T., \& Joubert, P. N. 1985, J. Fluid Mech., 157, 405

Yecko, P., \& Rossi, M. 2004, Phys. Fluids, 16(7), 2322 